\section{Inequities in neurology amplified by the COVID-19 pandemic}

\author{
LaShyra Nolen $\odot$ and Nicte I. Mejia@
}

\begin{abstract}
This year, the COVID-19 pandemic has altered neurological care in many ways. However, evidence indicates that people from marginalized ethnic and socioeconomic groups have been affected by these changes more than others, highlighting and amplifying existing health-care disparities.
\end{abstract}

During 2020, the coronavirus disease 2019 (COVID-19) pandemic negatively affected the health of many people, both directly and via an impact on health-care provision and access. However, these effects were not evenly distributed across all groups within society. A growing body of evidence indicates that the impact of the severe acute respiratory syndrome coronavirus 2 (SARS-CoV-2) has been magnified by poverty, racism, and their consequences, which include disparities in access to health care ${ }^{1}$. In this Year in Review, we discuss four publications that help us understand how the COVID-19 pandemic is highlighting and amplifying inequity in neurology.

The first key publication ${ }^{2}$ is a retrospective analysis of a cohort comprising 69 patients across the USA and Canada who experienced acute ischaemic stroke in the context of COVID-19 infection. This analysis found that the rates of symptomatic intracranial haemorrhage and death were significantly higher among patients who identified as Black than among those who identified as white, Asian, or Latino. The reason for this disparity is not completely clear. Although Black patients in the cohort were significantly more likely to have diabetes mellitus and elevated LDL levels than other patients, the rate of other stroke risk factors, as well as stroke severity at presentation, door-to-CT time and functional disability score did not differ between the two groups. One concerning observation was that Black patients had lower rates of receipt of thrombolysis and thrombectomy, raising the possibility that unequal access to therapeutics could underlie the observed outcome inequities.

A second retrospective cohort study ${ }^{3}$ published this year characterized the neurological manifestations, risk factors and clinical outcomes among 509 patients hospitalized with COVID-19 at an academic medical centre (AMC, tertiary care hospital integrated with a medical school) and nine other hospitals within a health system in Chicago, Illinois. Neurological manifestations, in particular encephalopathy, were observed in $82.3 \%$ of participants and were particularly common in those with severe COVID-19. On average, the patients hospitalized at the non-AMC hospitals had worse functional outcomes and greater 30-day mortality than patients hospitalized at the AMC hospital, despite having similar COVID-19 severity, fewer comorbidities and less frequent neurological manifestations. In addition, the patients at the non-AMC hospitals had a greater likelihood of having 'do not resuscitate', 'do not intubate' and 'transition to comfort measures only' orders and were described as having reduced access to intensive and specialty care.

A third retrospective cohort study analysed the effect of the start of the COVID-19 pandemic on telestroke care in a network covering 38 hospitals across South Carolina, $\mathrm{USA}^{4}$. The median number of weekly consultations was significantly lower in March and April 2020 than in the 12 months prior to the pandemic; the largest proportional decrease was for consultations for Black patients. This observation suggests that COVID-19 is preventing already underserved patients from accessing neurological care. Similarly, data from the National Syndromic Surveillance Program (NSSP) showed a $20 \%$ decline in emergency room visits among people experiencing acute stroke during the first 10 weeks of the pandemic ${ }^{5}$. Although analyses of the NSSP data by race were not published, a crosssectional web-based survey of 9,896 adults representative of the US population showed that emergency care avoidance because of COVID-19 related concerns was significantly higher among participants who identified as Black or Latino than among participants who identified as white ${ }^{6}$. Taken together, these findings suggest that the pandemic is limiting access to emergency care for Black and Latino populations specifically. This conclusion is concerning as evidence indicates that, compared with white patients, Black and Hispanic patients with neurological conditions are less likely to receive care from outpatient neurologists and instead rely more heavily on emergency departments?.

Another key publication from this year ${ }^{8}$ described the results of an international, cross-sectional, web-based survey of 5,378 people living with Parkinson disease without known COVID-19 infections. Participants reported that during the pandemic they had experienced disruptions in health care, social engagement and exercise, as well as a worsening of motor and non-motor symptoms. Participants with a lower household income were less likely to attend telemedicine appointments, had greater difficulties obtaining medications and were less likely to find alternative means of exercise than participants with a higher household income. The small number of study participants who identified as African American, Asian, American Indian, Alaska Native, Native Hawaiian or Pacific Islander also reported greater difficulties in obtaining medications than participants who identified as white. These findings suggest that the effect of the COVID-19 pandemic on people living with neurological conditions is greater for already marginalized and underserved groups, and highlight existing structural barriers to health care, for example, the digital divide, which limits the ability of patients to attend telemedicine appointments and communicate via patient portals ${ }^{9}$.

\section{Key advances}

- Black patients with acute ischaemic stroke and COVID-19 have faced higher morbidity and mortality than white, Asian or Latino patients ${ }^{2}$.

- Patients hospitalized with COVID-19 at non-academic medical centres had worse outcomes than those at academic medical centres; gaps in access to critical and specialty care might influence these disparities ${ }^{3}$.

- Black patients with acute ischaemic stroke were less likely than white patients to receive telestroke services during the pandemic; gaps in access to acute stroke care have also been observed among Latino patients ${ }^{5}$.

- Among patients with Parkinson disease, those with lower incomes have had worse access to health care during the pandemic than patients with higher incomes ${ }^{9}$. 
These publications indicate that the COVID-19 pandemic is a particular burden for Black, Latino and lower-income populations. Although we suspect this burden extends to Native American and other Indigenous communities, and that inequities occur beyond the USA, these topics remain largely ignored in the neurology literature. To eliminate the observed disparities, we must dismantle the structural barriers that prevent equal access to neurological care across racial, socioeconomic and geographical lines (FIG. 1). In our opinion, this requires recognition that the challenges stem from societal values

$$
\text { the challenges stem from societal values and implicit bias }
$$

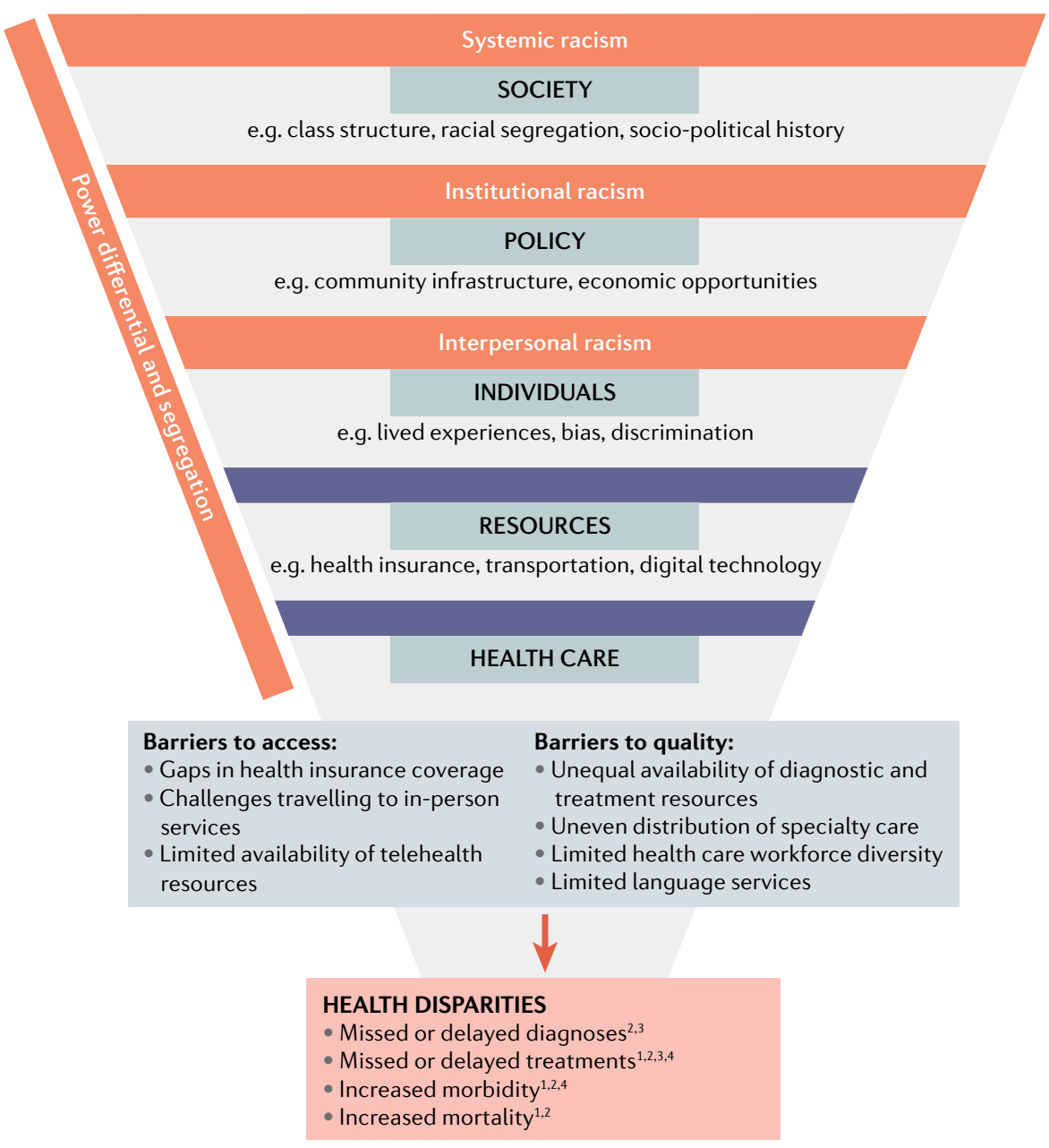

Fig. 1 | The factors that influence access to health care, receipt of quality neurology care and neurological outcomes as noted in the highlighted studies. Systemic, institutional and interpersonal racism all perpetuate segregation and prevent equitable access to resources, including quality health care, resulting in disparate health outcomes.

that perpetuate oppression and uneven power distribution delineated by social constructs like race, gender and class. Importantly, what happens in health care is intricately related to governance and public policies. Major factors that prevent people of colour and those of lower income from receiving health care include inequitable access to health insurance, gaps in access to technology and pervasive Even within the health-care system ${ }^{1,1,10}$. Even if patients of marginalized identities can surpass these barriers, they still face inequitable hospital policies, interpersonal racism and implicit bias ${ }^{10}$. These factors manifest as

\section{SOCIETY}

titutional racism

POLICY

HEALTH DISPARITIES
- Missed or delayed diagnoses ${ }^{2,3}$
- Missed or delayed treatments
- Increased morbidity ${ }^{1,2,3,4}$
- Increased mortality

Barriers to quality:

Unequal availability of diagnostic and

Limited health care workforce diversity

Limited language services disparities in neurological care, which have been amplified by the COVID-19 pandemic.

Health equity in neurology is possible, but we must first understand the systemic factors and pathways that underlie disparate health outcomes. As the COVID-19 pandemic evolves, the neurology community must measure, report and track disparities; work with health-care systems to implement structural changes that assure equal access to neurology care; advocate for public policies that facilitate universal access to health care; and engage with communities to combat structural racism and socioeconomic inequity. The year 2020 has made clear the work needed to improve the neurological health of some of our most vulnerable patients, now it's up to us to do it.

LaShyra Nolen (D)' and Nicte I. Mejia (iD ${ }^{1,2}{ }^{凶}$

${ }^{1}$ Harvard Medical School, Boston, MA, USA.

${ }^{2}$ Department of Neurology, Massachusetts General Hospital, Boston, MA, USA.

凶e-mail:nmejia@partners.org

https://doi.org/10.1038/s41582-020-00452-x

1. Metzl, J. M., Maybank, A. \& De Maio, F. Responding to the COVID-19 pandemic: the need for a structurally competent health care system. JAMA 324, 231-232 (2020).

2. Dmytriw, A. A. et al. Ischaemic stroke associated with COVID-19 and racial outcome disparity in North America. J. Neurol. Neurosurg. Psychiatry https://doi. org/10.1136/jnnp-2020-324653 (2020).

3. Liotta, E. M. et al. Frequent neurologic manifestations and encephalopathy-associated morbidity in Covid-19 patients. Ann. Clin. Trans/ Neurol. https://doi.org/ 10.1002/acn3.51210 (2020).

4. Cummings, C., Almallouhi, E., Al Kasab, S., Spiotta, A. M. $\&$ Holmstedt, C. A. Blacks are less likely to present with strokes during the COVID-19 pandemic: observations from the buckle of the stroke belt. Stroke 51, 3107-3111 (2020).

5. Lange, S. J. et al. Potential indirect effects of the COVID-19 pandemic on use of emergency departments for acute life-threatening conditions - United States, January-May 2020. MMWR 69, 795-800 (2020).

6. Czeisler, M. Ė. et al. Delay or avoidance of medical care because of COVID-19-related concerns United States, June 2020. MMWR 69, 1250-1257 (2020).

7. Saadi, A., Himmelstein, D. U., Woolhandler, S. \& Mejia, N. I. Racial disparities in neurologic health care access and utilization in the United States. Neurology 88, 2268-2275 (2017).

8. Brown, E. G. et al. The effect of the COVID-19 pandemic on people with Parkinson's disease. J. Parkinsons Dis. 10, 1365-1377 (2020).

9. Gray, D. M., Joseph, J. J. \& Olayiwola, J. N. Strategies for digital care of vulnerable patients in a COVID-19 world - Keeping in touch. JAMA Health Forum https:// doi.org/10.1001/jamahealthforum.2020.0734 (2020).

10. Bailey, Z. D. et al. Structural racism and health inequities in the USA: evidence and interventions. Lancet 389, 1453-1463 (2017).

Competing interests

The authors declare no competing interests. 